\title{
Avatars des déchets nucléaires japonais
}

Japanese Nuclear Waste Avatars

\section{Peter Wynn Kirby et Daniel Stier}

\section{(2) OpenEdition}

\section{Journals}

\section{Édition électronique}

URL : https://journals.openedition.org/tc/8112

DOI : $10.4000 /$ tc. 8112

ISSN : 1952-420X

\section{Éditeur}

Éditions de l'EHESS

\section{Édition imprimée}

Date de publication : 31 octobre 2016

Pagination : 356-369

ISBN : 9782713225291

ISSN : 0248-6016

\section{Référence électronique}

Peter Wynn Kirby et Daniel Stier, « Avatars des déchets nucléaires japonais », Techniques \& Culture [En ligne], 65-66 | 2016, mis en ligne le 31 octobre 2018, consulté le 29 septembre 2022. URL : http:// journals.openedition.org/tc/8112; DOI : https://doi.org/10.4000/tc.8112 


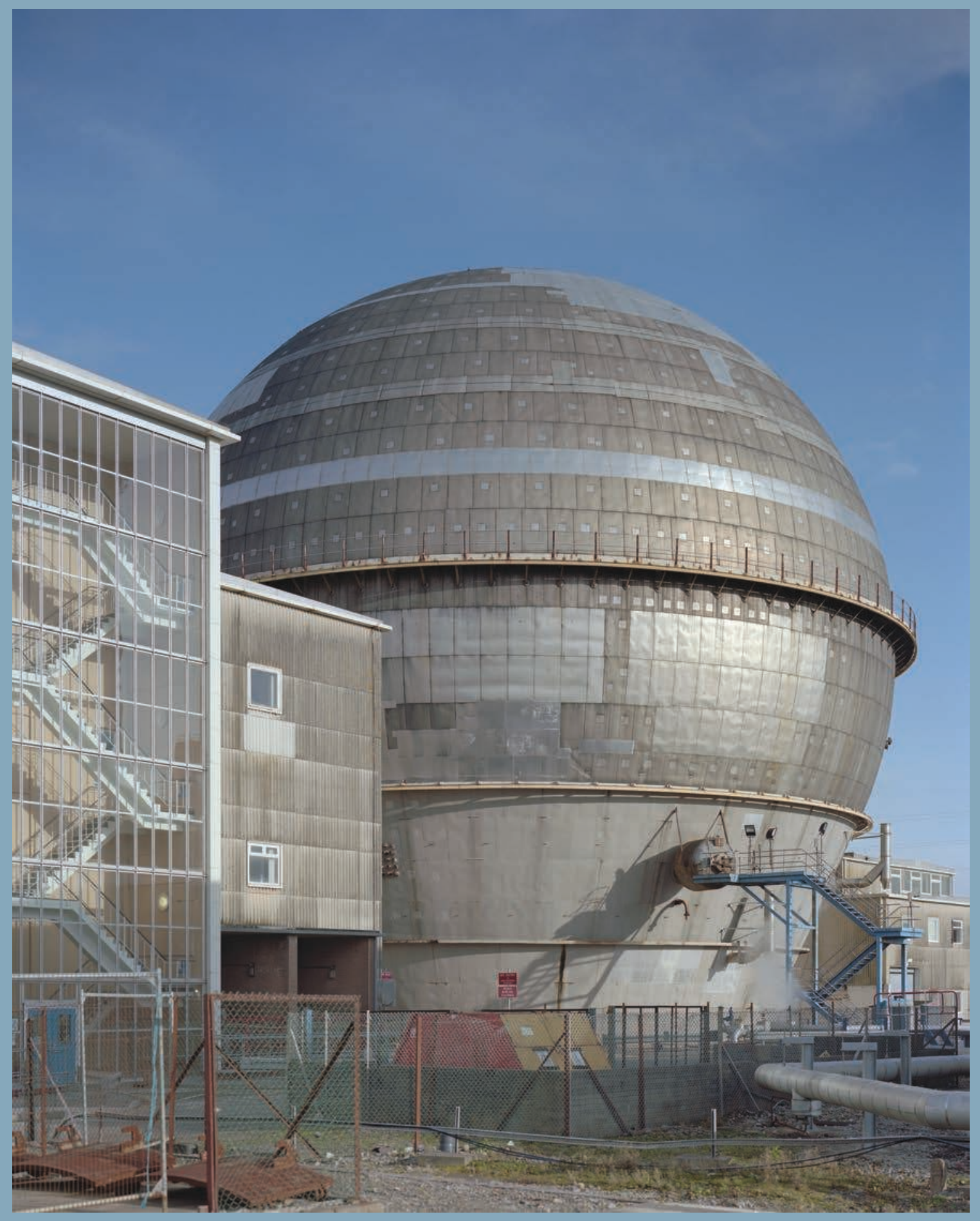




\section{Avatars des déchets nucléaires japonais}

Les déchets nucléaires ne donnent pas seulement des radiations alarmantes mais génèrent des échos ambivalents dans les discours qui oscillent entre l'exceptionnel et le banal (Hecht 2012). Alors que l'imagination populaire se représente des déchets nucléaires relativement immobiles, leur gestion requiert un ballet complexe impliquant de nombreuses étapes, des transformations, et du transport, franchissant fréquemment des frontières internationales. Cette instabilité géographique, combinée à la controverse sur les dangers des radiations nucléaires et à un manque obstiné de transparence dans l'industrie - héritage de programmes d'armement de la guerre entourés de secret- provoque un lourd artéfact culturel irradié dont la menace invisible correspond tout à fait à la rumeur et à l'anxiété qu'elle véhicule. Par conséquent, le spectre pernicieux des radiations atomiques plane de nouveau sur le Japon de l'après Fukushima, émergeant d'une période de latence induite par la propagande pour exploiter la crainte des radiations répandue chez les Japonais depuis 1945.

Dans ce contexte instable, le cataclysme japonais du tsunami de 2011 est devenu une vaste expérience indésirable de la gestion des déchets (pour paraphraser Blakemore 1976: 1). Tandis que les déchets nucléaires à haute radioactivité (HAVL: déchets de haute activité et à vie longue) vitrifiés constituent probablement la forme de déchets nucléaires la plus largement imaginée -et le Japon possède un énorme stock d'uranium et de plutonium dans une large gamme d'installations et d'avatars des déchets-, la centrale de Fukushima Daiichi elle-même et ses environs autrefois ordinaires ont pris la forme de déchets nucléaires encombrants. Ce n'est pas uniquement la chute du combustible nucléaire qui a fondu à travers l'enceinte du réacteur mais aussi tous les composants irradiés de la centrale et les environs chargés de décombres qui constituent un défi redoutable pour la gestion des déchets nucléaires. Cet article examine le spectre complet des déchets nucléaires du Japon post-tsunami japonais, depuis les barres de combustibles irradiées jusqu'à l'enceinte de confinement du réacteur déformé, et les façons dont les déchets nucléaires renvoient ou non à la manière de traiter les déchets quotidiens dans la culture japonaise. 
Le séisme et le tsunami de 2011 n’ont pas seulement apporté des déchets sur une grande partie de l'infrastructure côtière - mais ils ont surtout effacé les classifications ordonnées, soignées et rassurantes, et la prévisibilité géographique qui servaient de fondement à la gestion des déchets nucléaires ainsi que l'image de marque du Japon depuis les années soixante. La représentation des HAVL aseptisés et cloisonnés dans des enceintes de confinement relativement stables, a longtemps été sélective et trompeuse. Malgré de beaux schémas du cycle du combustible nucléaire, l'industrie nucléaire reste polluante - sans parler des émissions carbone - à toutes les étapes d'approvisionnement, d'exploitation, et d'élimination. Les déchets nucléaires de plus grande envergure, comme les HAVL, créent des problèmes de gestion particulièrement délicats. L'industrie nucléaire du Japon a longtemps été surnommée « une résidence sans sanitaires» (toire no nai manshon) pour son absence d'entrepôt définitif des déchets nucléaires. La politique autour de la recherche constante menée par le gouvernement pour une commune qui voudrait bien accueillir un site de stockage nucléaire a été intense et, de façon prévisible, épineuse dans la période post-2011. Même le récent accord du gouverneur de Fukushima d'accepter simplement un site de stockage temporaire pour les déchets radioactifs sur $16 \mathrm{~km}^{2}$ près des réacteurs endommagés, en septembre 2014, s'est heurté à une grande opposition publique et a coûté cher: plus de 300 milliards de yens, semble-t-il, en subventions d'État, loyers fonciers compris. Nombre de résidents de Fukushima exaspérés sont déterminés à faire en sorte qu'aucun entrepôt permanent ne finisse par être implanté dans leur province en difficulté.

Pourtant il existe une explication à l'absence de ces «sanitaires » pour uranium: l'élite obstinée du Japon a décidé, pendant des décennies, de transformer les résidus nucléaires en ressources, en retraitant par exemple les barres de combustible en nouveau combustible pour réacteurs. Depuis le début du $\mathrm{xx}^{\mathrm{e}}$ siècle, l'obsession des leaders japonais quant à la pénurie de ressources de l'archipel - invoquée couramment dans le discours sur le Japon comme étant une «petite nation insulaire, pauvre en ressources» (shigen shōkoku nippon) - a sans doute conduit à un militarisme d'avant-guerre agressif et à l'attaque de Pearl Harbor en 1941, parmi d'autres sombres évènements marquants (Sato 2007, Dinmore 2006). Néanmoins, la volonté du Japon d'après-guerre de garantir un accès sûr aux ressources énergétiques a continué comme si l'effet dévastateur de la période «transwar » n'était qu'un simple déficit en approvisionnement. L'enthousiasme de l'élite pour la construction de barrages et la production d'énergie hydroélectrique au Japon dans les années cinquante est passé habilement à la promotion active du nucléaire, qui a véritablement commencé dans les années soixante (Dinmore 2006, 2013). Dans ces arrangements, dans l'espace insulaire du Japon, politiquement et intellectuellement incestueux de «village nucléaire » (genshiryoku mura), comme on l'a baptisé, les déchets nucléaires n’ont jamais vraiment été considérés comme des « déchets», étant donné qu'ils pouvaient potentiellement être retransformés en combustible nucléaire supplémentaire. Les tentatives laborieuses du Japon pour mettre fin au cycle du carburant nucléaire se sont largement faites dans cet objectif.

Le rêve d'une énergie potentiellement inépuisable obtenue par la conversion des barres de combustible utilisées en un carburant à oxyde mixte (aussi connu sous le nom de MOX, un mélange d'uranium et de plutonium issu de combustible utilisé) a toujours plané de façon plus 
séduisante que la réalité inefficace et mal gérée. Le complexe de retraitement de Rokkasho au nord du Japon -cité par les critiques comme étant possiblement la structure civile la plus chère de l'histoire de l'homme (McCormack 2011) - a fonctionné depuis sa création en deçà des attentes ou pas du tout. Un retraitement domestique a également eu lieu à Tokaimura, situé à environ 125 km de Tokyo. Là-bas en 1999, un incident de «criticité », dans lequel des ouvriers peu formés ont sauté des étapes de sécurité pour verser de l'uranium liquide directement de seaux dans un bassin de décantation en concentration dangereusement élevée, est devenu l'accident nucléaire japonais le plus grave depuis 1945. Encore plus notoire, le surgénérateur Monju situé à Tsuruga, sur la Mer du Japon, a été conçu pour exploiter les résidus d'uranium et de plutonium - probablement substance planétaire la plus dangereuse créée par l'homme comme source d'énergie. Achevée en 1994, l'installation a été mise hors-service en 1995 quand son système de refroidissement au sodium a pris feu après une fuite. Après un camouflage raté, pendant plus d'une décennie de réparations, et, en 2010, un redémarrage manqué et un accident, l'installation Monju n'a produit qu'une infime quantité d'énergie durant ses vingt années d'existence. (Elle est maintenant susceptible d'être mise hors service). Toutes ces tentatives pour mettre fin au cycle nucléaire du Japon ont souffert du «mythe de la sécurité » répandu par l'industrie nucléaire qui a conduit à des régimes d'inspections mal conçues et mal mises en œuvre, à une maintenance négligée, à un excès de confiance envers des travailleurs embauchés en sous-traitance et manquant de compétences, et à une pensée unique généralement répandue.

Cependant, en dépit de l'histoire récente négligente voire bâclée de la gestion du nucléaire du Japon, la nation partage avec la majorité du reste du monde son insouciance délibérée à l'égard de la durabilité embarrassante des déchets nucléaires. Dans le calcul des coûts de l'énergie nucléaire et la viabilité de l'appareil nucléaire, peu de nations se préoccupent d'évaluer de façon responsable la nécessité institutionnelle, de sauvegarder des déchets à forte radioactivité sur des milliers d'années. Le calcul contesté des vrais coûts de la «nucléarité » au Japon se combine avec les répartitions géographiques inégales des déchets nucléaires. Le Japon, comme la France (Aldrich 2008), a choisi pendant des décennies des sites nucléaires pour des raisons qui dépassent les considérations scientifiques, par souci d'opportunisme politique et économique. Cette méthode a vu la plupart des installations nucléaires placées dans les zones du pays les plus pauvres, les plus rurales et les moins émancipées. Ce n'est pas un hasard si Fukushima Daiichi et les autres installations nucléaires ont été construites sur le territoire d'une préfecture historiquement ruinée et politiquement marginalisée - rien moins qu'une colonisation de l'arrière-pays ${ }^{1}$. (Après tout, l'énergie produite à Fukushima Daiichi n’alimentait pas les communes locales ni même la région mais allait directement de Fukushima à la capitale. Entre-temps, comme c'est désormais bien connu à l'échelle internationale, les risques associés à l'approvisionnement de l'énergie atomique sont tragiquement restés derrière). Ce n'est pas non plus particulièrement surprenant que l'on trouve la majeure partie de l'infrastructure japonaise des déchets nucléaires regroupée sur la péninsule de Shimokita, une des régions du pays les plus reculées socialement et géographiquement.

La péninsule de Shimokita, à l'extrémité la plus septentrionale de l'île principale de Honshū, hébergeant l'un des trois sites les plus sacrés du Japon, a longtemps fait l'objet 
Flasques de matière nucléaire japonaise, prêtes au transport vers

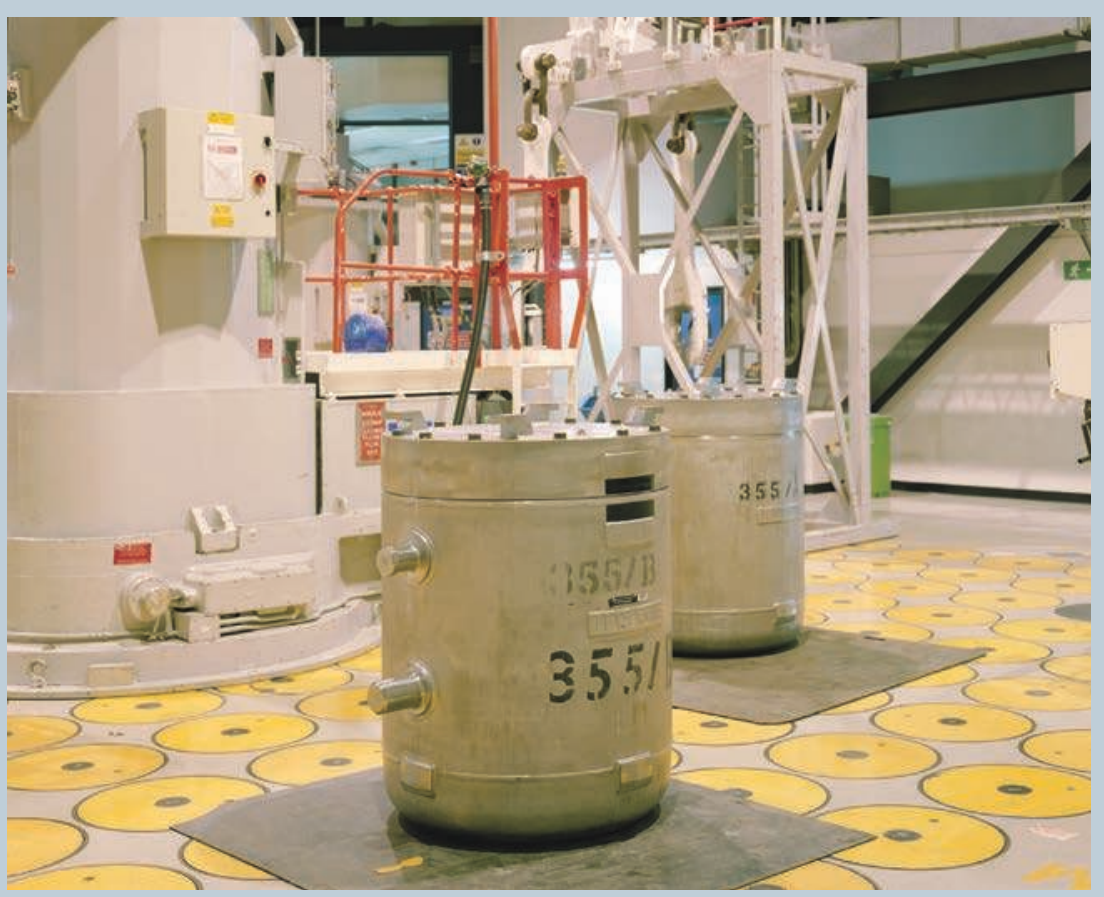

2. L'interieur de THORP, l'usine de retraitement de Sellafield, Royaume-Uni.

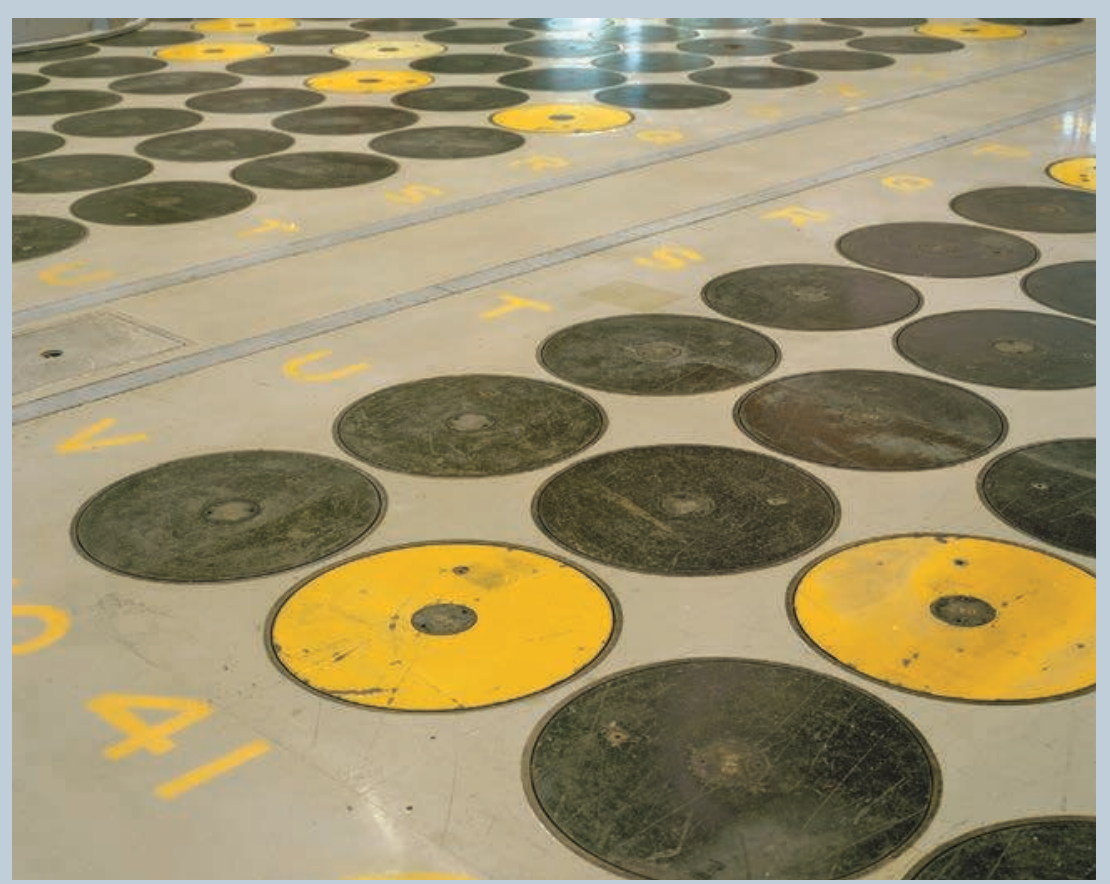




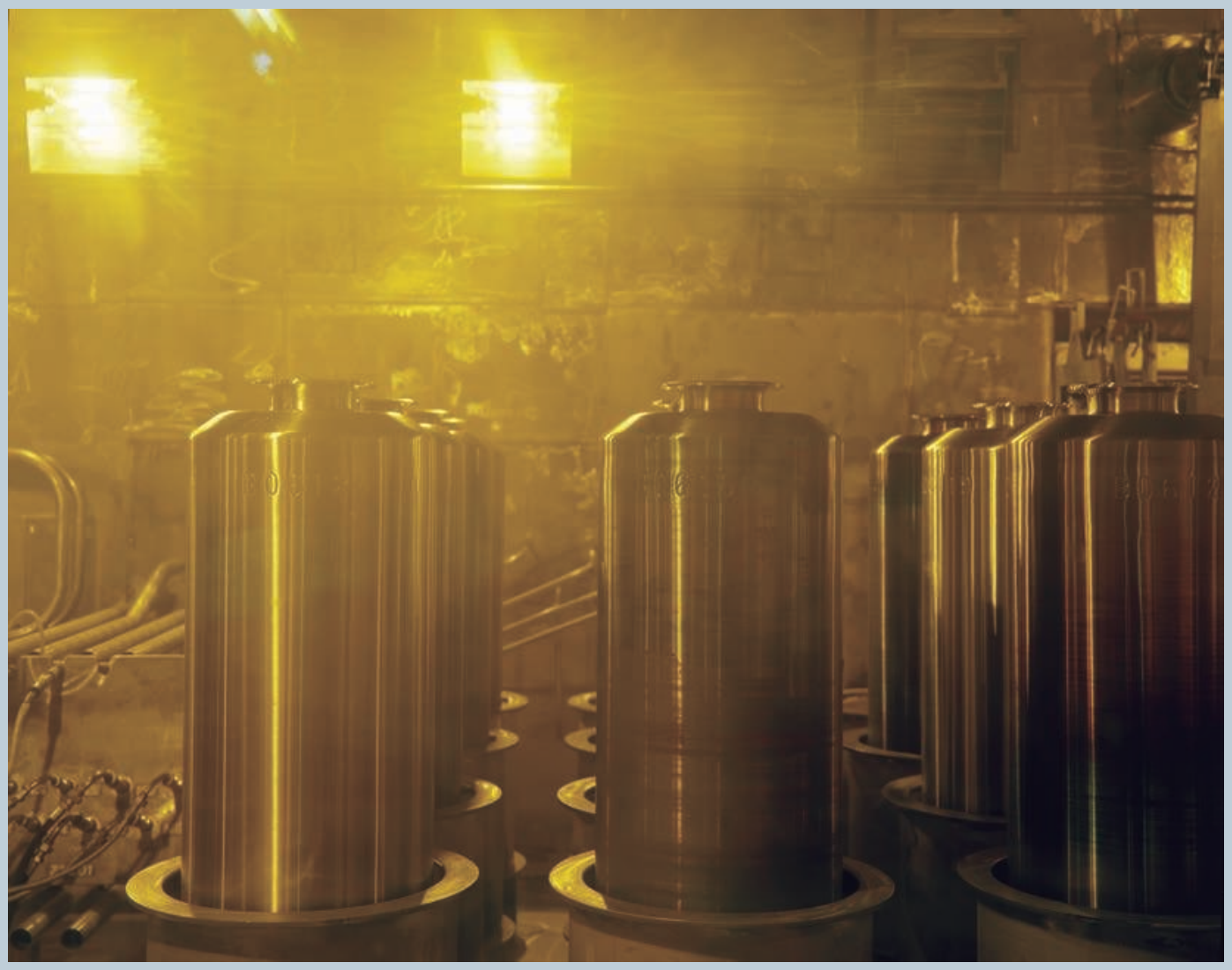

3. HAVL japonais à Sellafield, Royaume-Uni. 


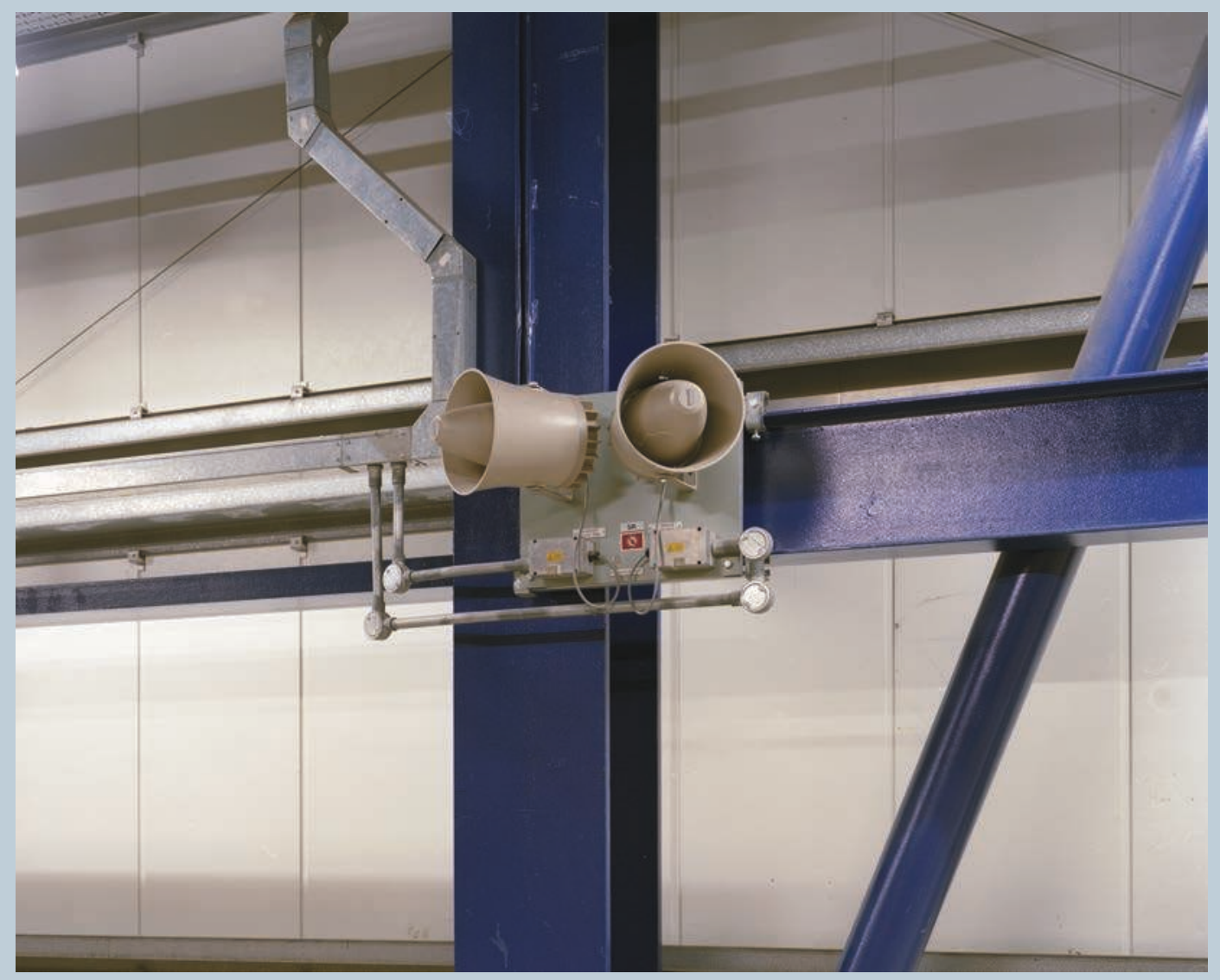

4. Sellafield, Royaume-Uni. 


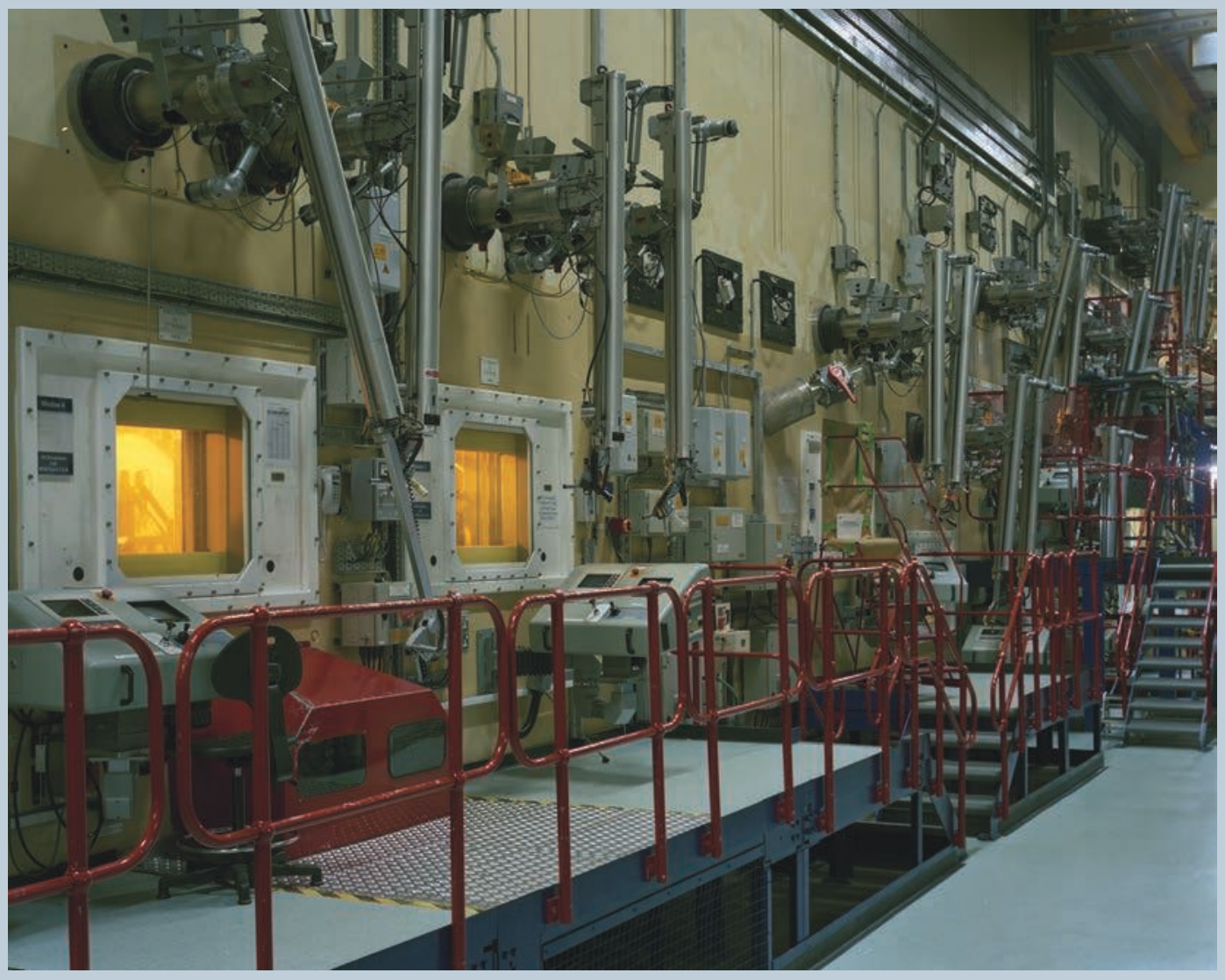

5. Sellafield, Royaume-Uni. 
d'une renommée spirituelle. Le paysage volcanique d'Osore-zan, ou «Mont de la peur», bouillonnant de gaz sulfureux, offre une représentation effrayante de l'enfer bouddhiste et rappelle aussi l'instabilité sismique et volcanique de l'archipel. Pourtant, cette péninsule géographiquement et culturellement très marginale accueille aussi deux des trois installations de stockage temporaire des déchets nucléaires de la nation, une à Rokkasho, et l'autre (encore provisoire) à Sekinehama. Ces installations - la seconde sous le slogan: « Nous les stockons correctement pour une vie future éblouissante $»^{2}-$ retraitent les déchets nucléaires et les stockent pour une utilisation dans le cycle du combustible nucléaire, à présent manifestement rompu. Le plutonium retraité sur la péninsule Shimokita était destiné à alimenter le réacteur Monju, aujourd'hui arrêté et ne peut être utilisé qu'en quantités relativement petites dans d'autres réacteurs au Japon. Le lobby japonais de l'énergie n'a toujours pas expliqué comment il envisage de répondre efficacement à ces graves problèmes.

Les stocks des déchets nucléaires s'étendent bien au-delà des limites maritimes de la nation. La stratégie énergétique du Japon centrée sur le plutonium a débouché sur un énorme stock d'environ 47 tonnes de plutonium, assez pour produire environ 6000 armes nucléaires, ainsi que des stocks considérables d'uranium. (Le Japon développe également un programme spatial abouti, qui lance des fusées ressemblant à des missiles, un fait qui n'est pas passé inaperçu chez ses rivaux susceptibles d'Asie de l'Est, la Chine et la Corée du Nord.) Cependant cette matière se trouve en grande quantité dans des sites de retraitement distants tels que THORP, une usine de retraitement thermique des oxydes, située à Sellafield, au Royaume-Uni. Le retraitement dans des installations comme THORP vise à séparer et raffiner l'uranium (environ 96\%) du plutonium (approximativement 1\%), les quelque 3\% de résidus restants étant traités et stockés. Le plutonium est transformé en combustible MOX, par exemple, et l'uranium converti en nouveau combustible lui aussi. En application de l'ambitieux calendrier de Sellafield, cette réserve satellite a été rapatriée à un rythme croissant. De telles exportations nucléaires ont été destinées à des dépôts de déchets intermédiaires présents sur la péninsule Shimokita. Mais avec l'équipement de l'usine de retraitement de Rokkasho établie pour produire $8000 \mathrm{~kg}$ de plutonium retraités par an, sans aucune capacité à proximité du réacteur pour prendre en charge de tels volumes, on ne sait pas au juste si l'interruption du cycle des déchets du Japon a créé un blocage en termes de capacité de stockage existant et de protocoles de retraitement.

Les évènements sismiques de mars 2011 ont créé une rupture fortuite embarrassante dans la pratique du nucléaire japonais, qui a exposé la gestion laxiste et problématique des déchets nucléaires dans ce pays ainsi que les distorsions dans sa conception même à un examen plus vaste. Négligeons l'usage rhétorique des termes «ressources» et «recyclage », la plupart des déchets nucléaires du Japon sont simplement transformés en d'autres formes de déchets. En des temps prospères et révolus, lorsque l'industrie nucléaire du Japon opérait avec plus de latitude, le processus qui a cours aujourd'hui impliquait de nombreuses étapes et un ballet complexe qui comportait de longues pérégrinations via bateau, camion et train. La gamme des avatars protéiformes formels et élémentaires que les déchets nucléaires japonais prennent habituellement - barres de combustible usé, boue radioactive, vitrification du borosilicate 
dans une enceinte en acier inoxydable, etc. - a augmenté infiniment suite à la destruction et à l'irradiation de Fukushima Daiichi et ses environs. L'infrastructure, celle-là même qui visait à protéger, isoler et contrôler la matière industrielle radioactive, a, au lieu de cela, échappé aux classifications pour entrer dans le flux des déchets non traités. En plus du combustible nucléaire qui a chuté et se trouve sous l'installation endommagée après les effondrements, l'enceinte de confinement tordue et, en fait, l'intégralité de la centrale de Fukushima Daiichi elle-même se sont transformées en une forme complexe de déchets nucléaires. Les usines doivent finalement être désaffectées - ce stade écologique et économique est rarement et à contrecœur inclus dans les calculs et les évaluations - mais le caractère peu méthodique et flou de l'installation et les radiations persistantes de la fusion compliquent grandement l'opération de nettoyage qui prendra vraisemblablement entre trois et quatre décennies.

En outre, les environs couverts de débris constituent un défi terrible relativement aux déchets nucléaires. De nombreux kilomètres carrés d'habitations, de routes, d'entreprises, de terre agricole et de forêt excèdent désormais le niveau maximum tolérable de radioactivité et nécessitent un assainissement. (Le gouvernement Abe continue de promettre aux personnes évacuées la restauration de leurs communes pour une future réintégration). Divers districts le long de la frontière de la zone d'exclusion ont été témoins des efforts d'assainissement naissants qui se multiplieront en d'autres endroits du terrain radioactif dépeuplé de la préfecture. Le programme de décontamination en cours a vu des millions de tonnes de terre et de matières végétales arrachées aux terrains scolaires, aux jardins, aux champs et à la couverture végétale de la forêt pour remplir des sacs plastiques, empilés sous des bâches dans des sites de stockage provisoires (kari-okiba), ou même des sites de stockage "provisoirement provisoires » (kari-kari-okiba). Les résultats d'un éventuel traitement ultérieur, controversé et pas encore confirmé incluent l'incinération massive des contenus combustibles de ces sacs empilés et/ ou leur répartition et leur transport vers des municipalités à travers la nation - ces dernières représentant une tentative japonaise caractéristique du «sacrifice commun». Coûtant des centaines de milliards de yens, la mesure d'assainissement de la surface colossale semble être un exercice futile, davantage une manifestation d'obsession de la pureté, emblématique des habitudes en ce qui concerne les déchets en général (Kirby 2011; voir aussi Douglas 1966, Ohnuki-Tierney 1984), qu'un projet réfléchi avec un résultat probablement positif. Dans le cas, cependant plausible, d'un succès des efforts de décontamination, il reste hautement discutable que la composante humaine volage puisse être restaurée à un quelconque niveau durable pour rendre véritablement ces communes à nouveau viables.

La centrale de Fukushima Daiichi demeure actuellement dans un état précaire d'«arrêt à froid »-témoignant régulièrement d'une stabilité modale mais restant, en ce cas, hautement vulnérable à une nouvelle activité sismique - grâce à des spaghettis de tuyaux d'eau élaborés pour maintenir les réacteurs de l'installation en dessous de $100^{\circ} \mathrm{C}$. Les immenses défis du site de Fukushima Daiichi - enceinte de confinement définitif en béton fissuré et enceinte de confinement de réacteur en acier ravagé et déformé - ont en premier lieu stimulé une série d'innovations impromptues. Et, ce qui est plus grave encore, ils requièrent un système de 
refroidissement improvisé qui génère des milliers de tonnes d'eau hautement radioactive laquelle doit être de préférence stockée plutôt que rejetée. Des tentatives pour filtrer les polluants n'ont pas vraiment fait l'unanimité; de façon notoire, le système TEPCO installé en juin 2011 pour filtrer le césium, le pétrole, et le sel de l'eau du circuit de refroidissement est tombé en panne dans les heures suivantes - les niveaux élevés de césium ont saturé le système. TEPCO est maintenant forcé de stocker l'eau contaminée sur son site; des réservoirs installés remplissent progressivement l'enceinte de $3,5 \mathrm{~km}^{2}$. Les écoulements souterrains des eaux venues des montagnes présentent aussi un casse-tête. Au moment où l'eau entre en contact avec le combustible nucléaire tombé sous l'installation, elle devient radioactive, et se jette ensuite dans l'océan en un volume estimé à plus de $265000 \mathrm{~L}$ par jour. TEPCO tente en 2015 de mettre en place une innovation sans précédent - enfonçant dans le sol, à intervalles réguliers autour de l'installation, d'énormes éléments de refroidissement ressemblant à des pylônes. Ceux-ci devraient, avec le temps, refroidir lentement le sol afin de créer une barrière thermique inférieure à $0^{\circ} \mathrm{C}$ qui empêcherait l'eau retenant les matières nucléaires tombées, d'entrer ou de sortir de l'enceinte. La technologie n'a pas été testée à cette échelle, et pourrait presque requérir un réacteur nucléaire qui répondrait aux énormes besoins en électricité, cependant le projet controversé reste en cours.

De nombreuses expressions qualifient les déchets du nucléaire parmi lesquelles «une ordure éternelle» (Zonabend 1993: 126) et «l'ultime déchet - détritus qui vont rester détritus très longtemps » (Hall 1996: 4). Dans une société qui a longtemps été obnubilée par le tri des ordures, le maintien de la pureté (imaginaire), et la gestion de proximité de la pollution (e.g., Kirby 2011) -sans parler du traumatisme des radiations atomiques de l'après 1945 auquel ils ont fait face-le spectre des déchets nucléaires, dans tous leurs avatars, plane sur le Japon contemporain et ses débats permanents sur les ressources, le risque, et l'identité nucléaire japonaise elle-même.

Cette discussion sur les déchets nucléaires et les risques nucléaires, éloignée de toute évidence des préoccupations quotidiennes de la population française plutôt plus pronucléaire, est devenue plus pregnante et pertinente suite à la déclaration ${ }^{3}$ de 2014 d'une coopération francojaponaise dans la 4e génération du réacteur rapide ASTRID, qui doit être construit sur le site de Cadarache en Provence-Alpes-Côte d'Azur, près d'Avignon. Paradoxalement, un fort intérêt français dans l'exploitation d'une technologie de la surgénération a créé la possibilité réelle de faire renaître l'installation Monju, comme Lazare, de ses restes atomiques défunts, au moins temporairement -apportant de cette manière un nouveau sens au nom «Superphénix » ${ }^{4}$. En voulant tester le réacteur Monju pour parfaire leur projet ASTRID - en particulier pour s'assurer que le système de refroidissement au sodium évite une répétition de la fuite de sodium désastreuse du réacteur japonais de 1995 et l'incendie qui s'ensuivit - les autorités nucléaires françaises risquent d'entacher leur propre bilan de sécurité avec Monju, secoué par les scandales.

Au moins depuis le débat télévisé présidentiel de 2007 entre Nicolas Sarkozy et Ségolène Royal, dans lequel les problèmes nucléaires en général et le destin du réacteur EPR d'Areva en particulier ont été brouillés dans un cocktail enivrant de politique, de vanité, d'ambition, de rhétorique nationaliste et de postures face aux enjeux environnementaux, le sort stratégique du 
vaste dispositif nucléaire de la France a pesé dans la balance. Avant la triple crise de Fukushima Daiichi en 2011, beaucoup de Français étaient fiers de l'indépendance énergétique de la nation (en termes de production électrique, au moins) et plus ou moins inconscients de tous les soucis de santé environnementale. Or aujourd'hui, la trajectoire de l'énergie de la France est devenue plus incertaine. L'aspiration très japonaise à un cycle nucléaire fermé et ordonné, avec un retraitement perpétuel des déchets de plutonium et d'uranium en carburant nucléaire, restera-t-elle la stratégie de l'énergie nationale de l'Hexagone à une période où se développent considérablement des énergies solaires, éoliennes et autres énergies fournies au niveau international (voir, par exemple, le texte stimulant de Keith Barnham [2014] intitulé La réponse brûlante: guide d’utilisateur de la révolution solaire)? Le CEA et le reste du «lobby» français du nucléaire continueront-ils à accueillir favorablement la technologie du surgénérateur rapide qui a mérité des critiques sur son coût, son manque de fiabilité, sa vulnérabilité, et son inefficacité? L'inertie technocratique œuvrant pour le maintien dans le nucléaire sera considérable mais on peut former l'hypothèse que le projet ASTRID, - la destruction continue de Fukushima Daiichi en toile de fond - aidera à tailler dans les discours une place qui soit favorable à un échange national sur les priorités énergétiques qui pourrait prendre en compte la capacité croissante des énergies renouvelables en Europe, envisager un large soutien complémentaire à des sources d'énergies vertes tout en traçant la voie à suivre pour la France.

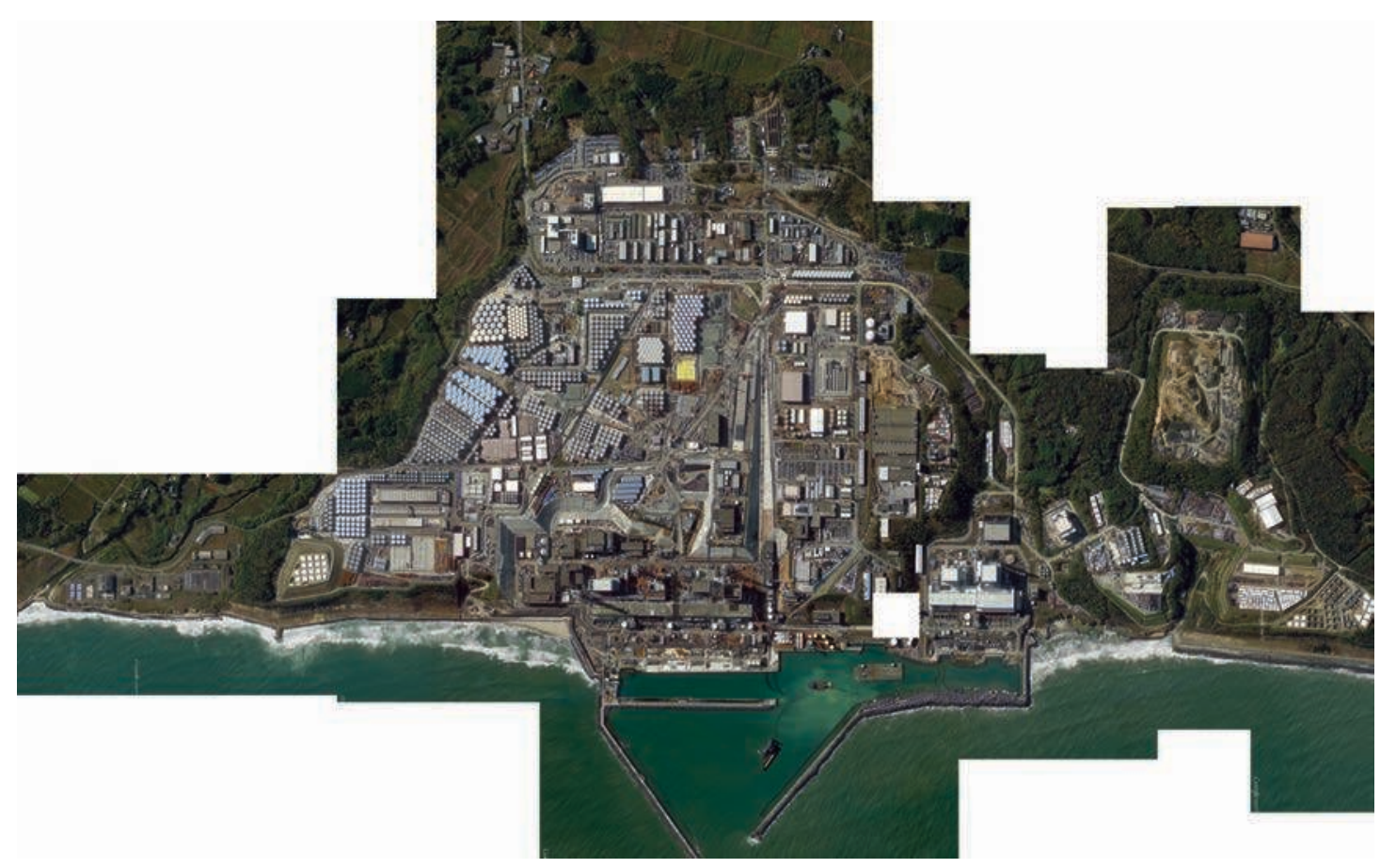

6. Image satellite du site de Fukushima Daiichi, empilement de réservoirs d'eau contaminée. 
«Dans un petit restaurant de la région de Tohoku, dans la ville convalescente de Ishinomaki, j'ai parlé en avril 2012 avec deux ingénieurs japonais qui avaient travaillé à la fameuse centrale Fukushima Daiichi le 11 mars 2011 quand le tremblement de terre record, et le tsunami monstrueux, ont déclenché le pire accident nucléaire au monde depuis Tchernobyl. Une telle rencontre en public aurait présenté un risque presque inimaginable pour des ingénieurs du nucléaire dans des communes du nucléaire étroitement surveillées, ce qui expliquait pourquoi ils avaient parcouru une bonne distance pour me rencontrer sur un terrain neutre, ce qu'ils considéraient comme plus sûr. L'un des ingénieurs était extraverti et loquace alors que l'autre restait visiblement méfiant, soucieux d'être vu et/ou entendu par des membres du «village nucléaire» (genshiryoku mura) du Japon, insulaire et tristement célèbre. Ils craignaient qu'être surpris à parler à un chercheur étranger puisse avoir des répercussions - une certaine variante contemporaine de mura hachibu, ou un bannissement traditionnel du village, pour ainsi dire. Bref, ils étaient inquiets du risque de perdre leur travail.

Matsui-san, le plus remuant, est finalement devenu très sérieux. "Ce que je veux vraiment dire, c'est que le Japon n'a simplement pas ce qu'il faut pour prendre en charge l'énergie nucléaire... Peut-être que si je parle de cela avec un étranger comme vous, quelque chose finira par changer.» Ignorant les avertissements étouffés de son ami, il a fustigé l'industrie nucléaire japonaise pour sa gestion de mauvaise qualité. «Mais, il n'y a pas que Fukushima Daiichi -je vois des problèmes tout le temps dans chaque réacteur que je visite, y compris dans la centrale nucléaire où je travaille actuellement [Fukushima Daini, située à $160 \mathrm{~km}$ au sud d'Ishinomaki] ». Son évacuation de justesse de Fukushima Daiichi, et les panaches irradiés qui continuent de sortir de l'installation, manifestement lui pesaient encore. Cette épave tordue reste aujourd'hui au premier plan de la politique de gestion des déchets nucléaires japonais.»

En aparté, récit et propos rapportés à l'auteur lors d'une rencontre à Ishinomaki, dans la région de Tohoku (Japon), en avril 2012.

\section{Notes}

1. Akasaka (2009) décrit l'ensemble de Tohoku comme une colonie interne exploitée pendant des décennies par les intérêts et la politique centrée sur Tokyo.

2. Bandeau de la page d'accueil de la compagnie (RFS): «Mirai no kurashi ga kagayakashiku yō niwatashitachi ga shikkari to chozō shimasu». (www. rfsco.co.jp). Cette formulation suit une ancienne stratégie qui associe la puissance nucléaire à des lendemains «brillants » ou «éblouissants » grâce à de fortes réserves d'électricité (ainsi que des subventions lucratives pour les communes d'accueil - voir Dusinberre 2012 : 116, 121-2).
3. La déclaration a été faite à un sommet au Palais de l'Élysée entre le premier ministre Shinzo Abe et le président François Hollande, le 5 mai 2014.

4. Superphénix, un réacteur surgénérateur à neutrons rapide, lui aussi, conçu par le CEA, a été mis en service en 1985 et a été fermé par Lionel Jospin en 1997. Les opinions concernant sa sécurité, son accessibilité financière, et son succès opérationnel en général varient radicalement. 


\section{Les auteurs}

Les travaux de l'anthropologue et ethnographe Peter Wynn Kirby portent sur l'identité du nucléaire japonais et la matérialité des déchets, et se fondent sur l'interaction directe avec les personnes concernées. Peter Kirby est chargé de recherches à l'École de Géographie de l'université d'Oxford. Avant d'intégrer cette dernière, il a consacré trois années à mener des recherches sur le nucléaire au Centre de recherches sur le Japon, EHESS, Paris. Outre la publication de deux livres et de nombreux articles universitaires, Peter Kirby diffuse ses recherches via la presse, dans le New York Times ou encore le Guardian.

Daniel Stier est un photographe installé à Londres. Son dernier volume, Ways of Knowing, a été publié en 2015 par YES Editions. www.danielstier.com.

\section{Iconographie}

Image d'ouverture. Sellafield, Royaume-Uni.

6. (c) Google Earth Pro.

\section{Références}

Akasaka, N. 2009 Tōhokugaku: Wasurerareta Tōhoku. (Études sur Tōhoku: Tōhoku oubliée) Tokyo: Kōdansha.

Aldrich, D. P. 2008 Site Fights: Divisive Facilities and Civil Society in Japan and the West. Ithaca, NY: Cornell University Press.

Barnham, K. 2014 The Burning Answer: A User's Guide to the Solar Revolution. Londres: Weidenfeld \& Nicolson.

Blakemore, C. 1976 «Lecture 3: An Image of Truth. Mechanics of the Mind», Reith Lectures. Broadcast 24 November. downloads.bbc.co.uk/rmhttp/radio4/ transcripts/1976_reith3.pdf.

Dinmore, E. 2013 «Concrete Results? The TVA and the Appeal of Large Dams in Occupation-Era Japan », The Journal of Japanese Studies 39 (1): 1-38.

-2006 A Small Island Nation Poor in Resources: Natural and Human Resource Anxieties in Trans-World War II Japan. Unpublished Ph.D. dissertation, Princeton University.

Douglas, M. 1966 Purity and Danger: An Analysis of the Concepts of Pollution and Taboo. Londres: Routledge \& Kegan Paul.
Crédits photographiques pour l'ensemble des images: (c) D. Stier

Dusinberre, M. 2012 Hard Times in the Hometown: A History of Community Survival in Modern Japan. Honolulu: University of Hawaii Press.

Hall, J. 1996 Real Lives, Half Lives: Tales from the Atomic Wasteland. Londres: Penguin.

Hecht, G. 2012 Being Nuclear: Africans and the Global Uranium Trade. Cambridge, Londres: MIT Press.

Kirby, P. W. 2011 Troubled Natures: Waste, Environment, Japan. Honolulu: University of Hawaii Press.

McCormack, G. 2011 «Hubris Punished: Japan as Nuclear State», The Asia-Pacific Journal 9, 16 (3).

Ohnuki-Tierney, E. 1984 Illness and Culture in Contemporary Japan: An Anthropological View. Cambridge: Cambridge University Press.

Sato, J. 2007 «Formation of the Resource Concept in Japan: Post-War Efforts in Knowledge Integration». Sustainability Science 2(2): 151-158.

Zonabend, F. 1993 The Nuclear Peninsula. Cambridge: Cambridge University Press.

\section{Pour citer cet article}

Kirby, P.W. \& D. Stier 2016 «Avatars des déchets nucléaires japonais », TechniquesECulture 65-66 « Réparer le monde. Excès, reste et innovation», p. 356-369. 